\title{
DIGITAL PROCESSING ON COMETS
}

\author{
D. KUBÁČEK, E.M. PITTICH and J. ZVOLÁNKOVÁ \\ Astronomical Institute, Slovak Academy of Sciences \\ Dúbravska cesta 9 \\ 84228 Bratislava \\ Slovak Republic
}

\begin{abstract}
Several digital techniques have been used to remove large-scale background variations caused by manufacturing and technology of the photographic processing on old photographic plates ORWO ZU-21. Suppression of these large-scale background variations improved the study of large-scale phenomena in cometary plasma tails and the determination of the solar wind characteristics.

The results obtained from five images of the comet Bradfield 1987 XXIX have been compared. It has been shown that the simple digital techniques used were applicable also to photographic plates with images of lesser quality. They may be utilized in the treatment of old photographic plate archives.
\end{abstract}

\section{Introduction}

In the past all large-scale observations of comets were performed on photographic plates. Many of them were exposed on emulsions which were not homogeneous. These plates have background variations caused by manufacturing and technology of the photographic processing. In spite of this drawback, the plates contain a large amount of historical information about comets. Therefore, we tried to select procedures which would remove or, at least lower these errors of emulsion to an acceptable limit.

For our experiment, we used five large-scale exposures of the comet Bradfield 1987 XXIX, taken between November 15 and December 23, 1987. The observations were obtained with the $63 / 85 / 187 \mathrm{~cm}$ Maksutov telescope of the Klet' Observatory. The comet was exposed on the ORWO ZU-21 plates $(13 \times 18 \mathrm{~cm})$. The plates were developed with contrast negative Fomadon $R$ for 4 minutes at $20^{\circ} \mathrm{C}$. The field-of-view was $4.5^{\circ} \times 3.5^{\circ}$.

\section{Observed Data and Plate Background Removal Techniques}

The plates were digitized at 8-bits per pixel with an $85 \mu \mathrm{m}$ pixel size. Because all selected plates had been exposed without a photometric wedge and the procedure for calibration of photographic plates using brightness profiles of field stars by Kormendy (1972) was inapplicable in our cases, no corrections were made for characteristic curve (conversion of photographic densities to relative intensities); in our experiments we used relative density levels instead of relative intensities.

There are many approaches to image enhancement that have been applied to study comets 
(Matuska et al. 1978; Klinglesmith 1981; Larson 1986; Larson \& Sekanina 1984a, 1984b, 1985; Djorgovski \& Spinrad 1985; Schwartz et al. 1989; Larson 1991). Much useful information can be found also in papers dealing with image processing of galaxies (Arp \& Lorre 1976; Lorre 1978; Djorgovski 1985; Schweizer \& Ford 1985; Walterbos \& Kennicutt 1985). In almost all cases, one of the most important steps in preparing the image data for later analysis is the precise determination of the background. Depending on the complexity of the input data and on the object of interest, different methods can be used to estimate and subtract the background, so as to eliminate its non-uniformity and large-scale variations. Many new or modified approaches devoted to these problems published recently, e.g. in a series of the ESO/ST-ECF Data Analysis Workshops, have already been implemented into the large image processing and data analysis software packages, such as IRAF, MIDAS and DAOPHOT. A very simple, but good, method for removing a slowly varying background, e.g. when a comet is projected on the Milky Way or some gaseous nebulae, taking advantage of a reference plate of the same field of the sky exposed at different epoch, was proposed by Fukushima \& Watanabe (1991). This method is very useful for the study of large scale phenomena in cometary plasma or dust tails. Due to absence of the reference plates for our set of images, we could not test this method. We focused on the methods of elimination of large scale background variations based on:

1) the local histograms (Thonnat \& Llebaria 1981);

2) two-dimensional high pass large median (Lorre 1978; Heasley 1984), mean, boxcar filtering;

3) the smearing of the input data with a broad Gaussian kernel (Frieden \& Wells 1978; Heasley 1984);

4) fitting a two-dimensional low-order polynomials (Watanabe et al. 1989) in the areas without the cometary image;

5) some kind of an unsharp masking (Auriere \& Coupinot 1989);

6) the method proposed by Djorgovski (1985) using a squeezing of the input image data, different smoothing techniques, and finally re-expanding smoothed image to the original size either with a spline or a bilinear interpolation.

\section{Creation of Background Mask}

The first step in our tests was to create a mask from the original data (Fig. 1, UL) in which the area containing the comet is excluded for further computations (we abbreviate the quarters of the figures as UL - upper left, UR - upper right; BL - bottom left, and BR - bottom right). A local background in each image was determined under the assumption that a maximum of a histogram computed for several small submatrices of $20 \times 20$ pixels represents the most common pixel density level - sky or a background value. Then the original image was clipped to a mean sky value. The resulting image creates the mask with a very small range of density levels, with the minimum equal to zero (or near zero) and the maximum equal to the sky value (Fig. 1, UR). In this way the computed mean sky value serves as the simplest estimate of the background, but does not remove completely its large-scale variation (Fig. 2, UL).

The models of the plate background were estimated from both the original image data and the masks, using the methods mentioned above. For determination of the sky background from the original data, using the scanning two-dimensional median (mean, boxcar) filter, the median window size should be greater than twice the size of the point spread function or a cross-section of the studied object, e.g. comets with their plasma/dust tail, which is not to be considered in 


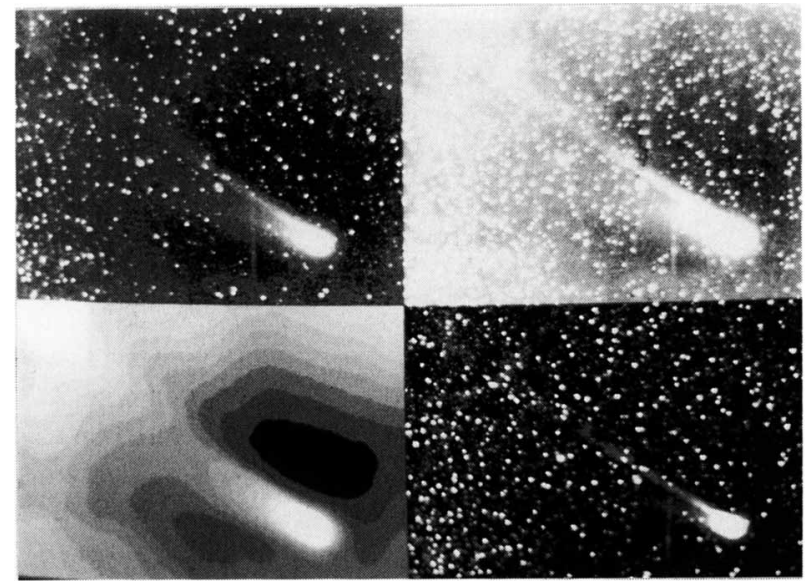

Figure 1. The original image data of comet Bradfield 1987 XXIX on November 25.73773, 1987 (upper left). The mask created from the original image using the local histograms; upper bound clipped to the mean sky value (upper right). The background model created from the mask using the large window median filter (bottom left). The foreground object is the result of the subtraction of the median background model from the original image and lower bound clipped to zero. The large-scale variation is removed (bottom right).

computations (Lorre 1978; Heasley 1984). It means that the filter size should be much larger than any of the object of interest and much smaller than the background component which is to be removed.

The median (mean, boxcar) window size is not so critical for a background model created from the mask. We used several window sizes from $11 \times 11$ to $31 \times 31$ pixels. For a background model created by smearing the original data with a broad Gaussian kernel, it is recommended to convolve the image data with the Gaussian kernel of standard deviation of threesigma of the point spread function (Frieden \& Wells 1978). In our case the FWHM of the point spread function on selected plates varied from 0.76 to 1.05 pixels, so sigma of the point spread function varied from 0.32 to 0.45 pixels. In our tests we used not three-sigma, but a five-sigma value.

Another type of background was created by fitting a bicubic spline over the mask. Some results of our tests can be seen in Figs. 1 - 4. In Fig. 1 in UL is the original image, in UR is the mask, BL represents the background model created from the mask using the median filter of $21 \times 21$ pixel size, and in BR we can see the foreground object as the result of subtraction of the background model from the original image and then clipped to zero.

In Fig. 2 some of the methods used in our tests are compared. In UL there is the image of the comet obtained simply by subtracting the mean background value produced from local histograms. UR represents the same image as BR in Fig. 1. BL in Fig. 2 illustrates the foreground image created by smearing the mask with a broad Gaussian kernel; and in BR of the same figure the result of a fit (interpolation) of a bicubic spline over the mask is shown.

Figure 3 represents the same images as in Fig. 2, but some details in foreground comets are enhanced by a histogram equalization. 


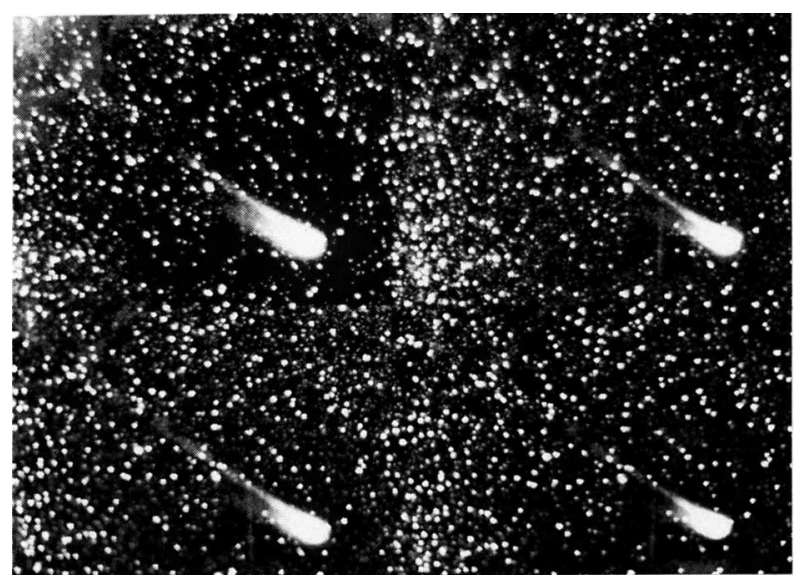

Figure 2. The foreground object after the subtraction of the computed mean background value using the local histograms. The large scale variation is not completely removed (upper left). The same as in Fig. 1 , on the bottom right (upper right). The large-scale variation of the plate eliminated by the subtraction of the background model computed by the convolution of the broad Gaussian with the mask (bottom left). The foreground object after subtraction of the background obtained by the fitting of bicubic spline over the mask (bottom right). In all pictures the lower bound less than zero was clipped to zero.

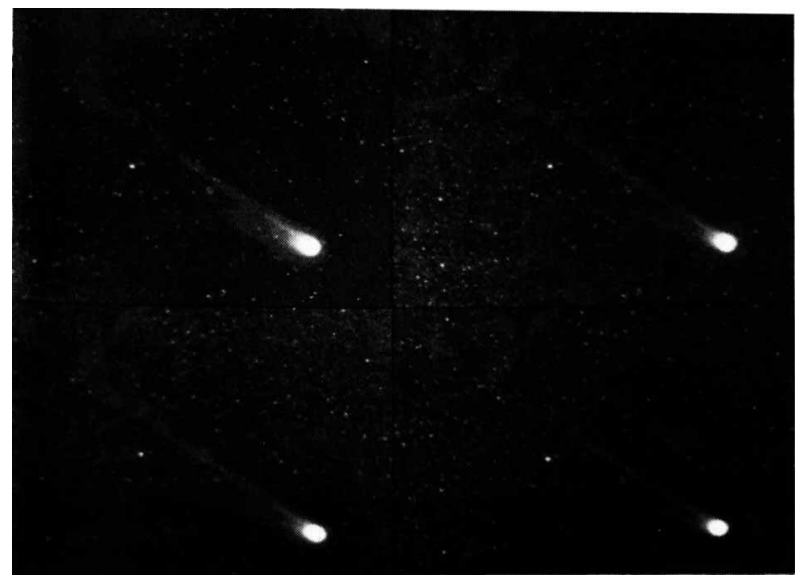

Figure 3. The same as Fig. 2 but some low-contrast features enhanced with histogram equalization.

In Fig. 4 the models of the background estimated by local histograms (UL), median filtering (UR), smearing with a broad Gaussian (BL), and fitting with a bicubic spline (BR) are compared. It should be noted that median filtering is a non-flux-preserving process, so the result of filtering the background should be slightly scaled before subtracting from the original data. 


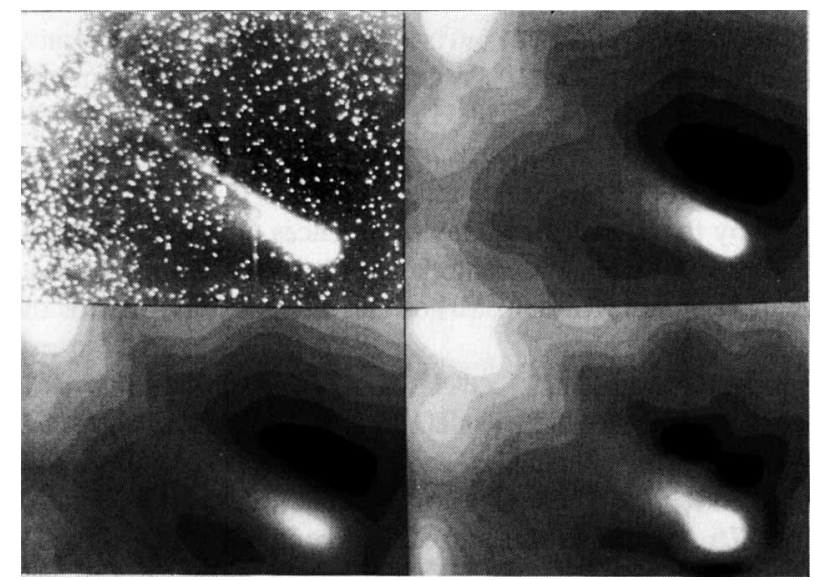

Figure 4. The mask as in Fig. 1, upper right (upper left). The median background model (upper right). The background model as a result of convolution of the broad Gaussian kemel with the mask (bottom left). Background estimated by a fitting of bicubic spline over the mask (bottom right).

The method of removing of the background, proposed by Djorgovski (1985), gives results similar to the median (mean) filtering or a Gaussian smearing, but is more demanding on computation capabilities. As a last test, we used a method proposed by Auriere \& Coupinot (1989). This unsharp masking enhances some details in the comet. This is very useful, e.g. for study of plasma tails, but does not remove a non-uniformity of the background completely. Removing a background completely and at the same time enhancing some details in the head or in a plasma tail of comet is possible also through directional gradients. In this way one can get nice 3-D pseudo-maps.

\section{Conclusions}

On five selected images of the comet Bradfield 1987 XXIX, which show the bright and long plasma tail, we have tested a number of image processing techniques useful for estimating and removing a slowly varying plate background. These methods are valuable not only for determination of the plate background, but also for later qualitative studies of cometary plasma tails.

According to our experience, the median window filtering gives better results for deriving the plate background than blurring the image with a broad Gaussian kernel or fitting methods, because both the latter lead to a halo around the bright features, e.g. a star or a head of a comet.

We have created the mask using the local histograms. However, the mask and the size of the median (mean) window or FWHM of a Gaussian kernel can be chosen arbitrarily according to the features of the object to be studied. The method described by Djorgovski (1985) gives results of a similar quality as the median filtering, but is computationally more demanding. The last tested method of unsharp masking (Auriere \& Coupinot 1989) enhances some small features in the plasma tail, but does not remove the large-scale variation of the plate background completely. 
All images were processed and displayed on the IBM/PC-compatible using software written by one of the authors.

\section{Acknowledgements}

This work was supported by the Slovak Academy of Sciences Grant No. 492/1993. The authors thank Jana Ticha, director of the Klet' Observatory, for kindly providing the plates, Milos Tíchy for the assistance at their selection, Antonín Mrkos and Zdenka Vavrova for their observations of the comet.

\section{References}

Arp, H. and Lorre, J.J., 1976. 'Image Processing of Galaxy Photographs', Astrophys. J., 210, 58. Auriere, M. and Coupinot, G., 1989. 'Background subtraction in the case of crowded fields and steep gradients', in 1st ESO/ST-ECF Data Analysis Workshop, eds. P. Grosbøl, F. Murtagh and R.H. Warmels, Garching, p. 101.

Djorgovski, S., 1985. 'Enhancement of features in galaxy images', SPIE Vol. 627, Instrumentation in Astronomy VI, p. 674.

Djorgovski, S. and Spinrad, H., 1985. 'Surface photometry of comet P/Encke', Astron. J., 90, $5,869$.

Frieden, B.R. and Wells, D.C., 1978. 'Restoring with maximum entropy. III. Poisson source and backgrounds', J. Opt. Soc. Am., 68, 1, 93.

Fukushima, H. and Watanabe, J., 1991. 'An image processing technique for background subtraction and its application to comet Austin 1989cl.' Publ. Natl. Astron. Obs. Japan, 2, 185.

Heasley, J.N., 1984. 'Numerical Restoration of Astronomical Images', P.A.S.P., 96, 767.

Klinglesmith, D.A., 1981. 'The interactive astronomical data analysis facility - image enhancement techniques applied to Comet Halley', in Modern Observational Techniques for Comet, JPL Publication 81-68, p. 223.

Kormendy, J., 1972. 'Calibration of direct photographs using brightness profiles of field stars', Astron. J., 78, 3, 255.

Larson, S.M., 1986. 'A review of some digital image processing in cometary research', in 'Asteroids, Comets, Meteors II', eds. C.-I. Lagerkvist, B.A. Lindblad, H. Lundstedt, and H. Rickman, Uppsala University, Uppsala, p. 449.

Larson, S.M., 1992. 'Evaluating some computer enhancement algorithms that improve the visibility of cometary morphology', in 'Asteroids, Comets, Meteors 1991', eds. A.W. Harris and E. Bowell, p. 337.

Larson, S.M. and Sekanina, Z., 1984a. 'Coma morphology and dust-emission pattern of periodic comet Halley. I', Astron. J., 89, 4, 571.

Larson, S.M. and Sekanina, Z., 1984b. 'Coma morphology and dust-emission pattern of periodic comet Halley. II', Astron. J., 89, 9, 1408.

Larson, S.M. and Sekanina, Z. 1985. 'Coma morphology and dust-emission pattern of periodic comet Halley. III', Astron. J., 90, 5, 823.

Lorre, J.J., 1978. 'Enhancements of the jets in NGC 1907', Astrophys. J., 222, L99. 
Matuska, W. Jr., Janney, D.H., Farrell, J.A. and Keller, C.F. Jr., 1978. 'Enhancement of Solar corona and comet details', Optical Engineering, 17, 6, 661.

Schwartz, G., Cosmovici, C., Mack, P. and Ip, W., 1989. 'Image processing techniques for gas morphology studied in the coma of comet Halley', Adv. Space Res., 9, 3, 217.

Schweizer, F. and Ford, W.K. Jr., 1985. 'Fine structure in elliptical galaxies', in 'New Aspects of Galaxy Photometry', ed. J.-L. Nieto, Lecture Notes in Physics, 232, Springer-Verlag, New York, p. 145.

Thonnat, M. and Llebaria, A., 1981. 'Two algorithms used to determine the plate background and applications', in 'Astronomical Photography 1981', eds. J.-L. Heudier and M.E. Sim, CERGA, Nice, p. 251.

Walterbos, R.A.M. and Kennicutt, R.C. Jr., 1985. 'Photographic surface photometry of the Andromeda Galaxy', in 'New Aspects of Galaxy Photometry', ed. J.-L. Nieto, Lecture Notes in Physics, 232, Springer Verlag, New York, p. 245.

Watanabe, J., Aoki, T., Taniguchi, Y. and Tarusawa, K., 1989. 'Photographic Observations of comet Bradfield 1987 XXIX at the Kiso Observatory', Publ. Natl. Astron. Obs. Japan, $1,71$. 